\title{
A Novel Flow-Perfusion Bioreactor Supports 3D Dynamic Cell Culture
}

\author{
Alexander M. Sailon, Alexander C. Allori, Edward H. Davidson, Derek D. Reformat, \\ Robert J. Allen Jr., and Stephen M. Warren
}

The Institute of Reconstructive Plastic Surgery Laboratories, New York University Medical Center, New York, NY 10016, USA

Correspondence should be addressed to Stephen M. Warren, stephen.warren.md@gmail.com

Received 18 March 2009; Revised 9 August 2009; Accepted 16 September 2009

Recommended by Chung-Liang Chien

Background. Bone engineering requires thicker three-dimensional constructs than the maximum thickness supported by standard cell-culture techniques $(2 \mathrm{~mm})$. A flow-perfusion bioreactor was developed to provide chemotransportation to thick (6 mm) scaffolds. Methods. Polyurethane scaffolds, seeded with murine preosteoblasts, were loaded into a novel bioreactor. Control scaffolds remained in static culture. Samples were harvested at days 2, 4, 6, and 8 and analyzed for cellular distribution, viability, metabolic activity, and density at the periphery and core. Results. By day 8, static scaffolds had a periphery cell density of $67 \% \pm 5.0 \%$, while in the core it was $0.3 \% \pm 0.3 \%$. Flow-perfused scaffolds demonstrated peripheral cell density of $94 \% \pm 8.3 \%$ and core density of $76 \% \pm 3.1 \%$ at day 8 . Conclusions. Flow perfusion provides chemotransportation to thick scaffolds. This system may permit high throughput study of 3D tissues in vitro and enable prefabrication of biological constructs large enough to solve clinical problems.

Copyright () 2009 Alexander M. Sailon et al. This is an open access article distributed under the Creative Commons Attribution License, which permits unrestricted use, distribution, and reproduction in any medium, provided the original work is properly cited.

\section{Introduction}

The replacement of tissue lost through trauma, disease, or congenital anomalies is a continuing clinical challenge. Current reconstructive options, including autologous tissue transfer, allograft, xenograft, and alloplastic implantation, are limited by donor site morbidity, tissue scarcity, disease transmission or antigenic incompatibility, hardware infection, and implant extrusion. Ideally, tissue reconstruction should avoid sacrificing healthy tissue or using alloplastic materials by instead engineering autologous replacement tissue de novo.

Tissue engineers have successfully cultured the cellular constituents necessary to build a variety of tissue types in vitro [1]. However, traditional two-dimensional (2D) cellculture techniques (e.g., Petri dishes and culture flasks) are inadequate for three-dimensional (3D) tissue engineering. In $2 \mathrm{D}$ culture, a monolayer of cells is in continuous contact with culture medium, and simple diffusion is sufficient to maintain cell viability [2]. As scaffolds gain $3 \mathrm{D}$ volume, however, the central core becomes increasingly separated from the penumbra of fresh medium; simple diffusion provides inadequate oxygen delivery and waste removal from cells in the core. As a result, only cells in a thin construct (with a large surface area-to-volume ratio) survive, and typically only on the peripheral crust of the scaffold (up to $2 \mathrm{~mm}$ deep) [3]. Nature has addressed this problem in native bone by establishing a complex lacunocanalicular network within which a nutrient-rich fluid circulates $[4,5]$. Thus, successful engineering of thick 3D osseous tissue constructs large enough to solve actual clinical problems will require novel tissue-engineering strategies that address chemotransportative requirements in their design and implementation.

The last decade has seen numerous attempts at improving chemotransportation for 3D constructs. For example, cell-seeded porous scaffolds have been set upon orbital shakers, hung in spinner flasks $[6,7]$, continuously perfused through glass columns [8], or tumbled in rotational bioreactors $[6,9,10]$. These methods increase medium fluid flow across the external surface of the scaffold, offering an incremental improvement over traditional static culture techniques. While these technologies satisfy the external 


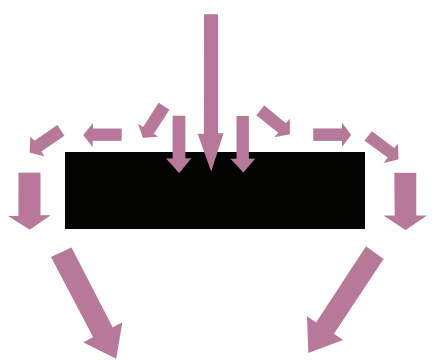

(a)

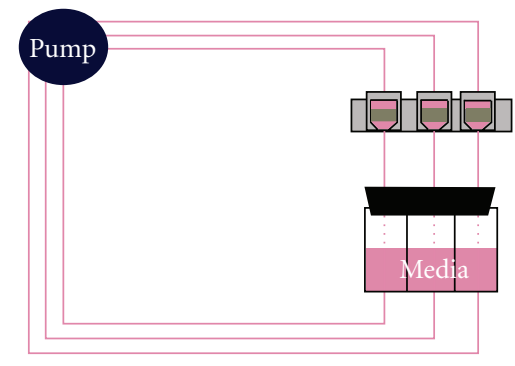

(c)

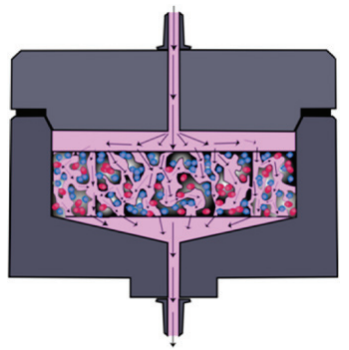

(b)

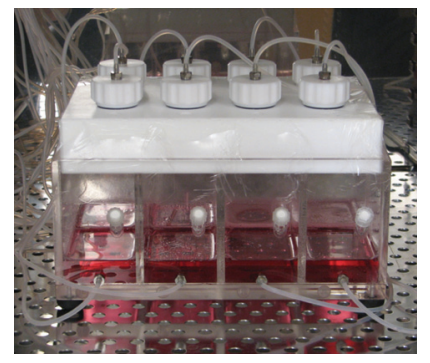

(d)

FIGURE 1: (a) With increased medium fluid flow across the external surface of the scaffold, chemotransportation is not guaranteed within the porous confines of the scaffold interior. (b) Schematic chamber within the flow-perfusion bioreactor. Note that medium is forced to percolate through the scaffold interior, ensuring chemotransportation to cells within the core. (c) Schematic of the 8-chamber flow-perfusion bioreactor within a standard cell culture incubator. (d) Photograph.

requirement for medium flow, convection of medium at the external surface does not guarantee chemotransportation within the porous confines of the scaffold interior [11]. In fact, the majority of medium in the systems described above follow the path of least resistance and circumnavigate the scaffold (Figure 1(a)) [3]. Consequently, convection of medium alone does not result in penetrating flow that perfuses the porous construct to provide effective chemotransportation.

A more promising idea for effective chemotransportation is poroelastic fluid flow [11]. In contrast to technologies like the spinner flask, we designed a flow-perfusion bioreactor to address the internal requirement for flow within the porous network of the scaffold (Figures 1(b) and 1(c)). In this system, porous scaffolds are press fit into an experimental chamber, and medium flows by gravity head or by generated hydrostatic pressure through the scaffold. Because the fluid path is confined to pass through the scaffold-none is lost to nonperfusing flow-the flow-perfusion bioreactor promises improved chemotransportation to all regions of a $3 \mathrm{D}$ scaffold. Furthermore, the flow-perfusion bioreactor is, in theory, a scalable technology that should support porous scaffolds of any thickness.

Nevertheless, to date, most exogenous tissue-engineering research has been constrained to using scaffolds $2 \mathrm{~mm}$ in thickness or less, which are readily sustainable by medium convection or static culture methods. Therefore, we evaluated the efficacy of a novel flow-perfusion bioreactor in sustaining "thick" 3D scaffolds that approach sizes of clinical relevance. Specifically, as proof of principle, we tested cylindrical scaffolds measuring $24 \mathrm{~mm}$ in diameter and $6 \mathrm{~mm}$ in thickness. Since these scaffolds are thicker than the "critical depth" of $2 \mathrm{~mm}$ (from core to surface), without effective fluid flow, they should suffer central core necrosis. We hypothesized that dynamic cell culture with a flow-perfusion bioreactor will provide adequate chemotransportation to the core of a thick scaffold, thereby, maintaining cell viability and activity.

\section{Materials and Methods}

2.1. Flow-Perfusion Bioreactor Design. The flow-perfusion bioreactor was machined from solid Teflon (SABIC Polymershape; Jacksonville, FL). It contains 8 independent experimental chambers each measuring $24 \mathrm{~mm}$ in diameter and able to accommodate scaffolds up to $10 \mathrm{~mm}$ in thickness (Figures 1(c) and $1(\mathrm{~d})$ ). The floor of each experimental chamber is tapered to ensure flow from the outer edges of the scaffold as well as the center to the exit port of the chamber (Figure 1(b)). Screw caps are fitted with Viton-75 O-rings (McMaster-Carr; Aurora, $\mathrm{OH}$ ) to ensure a tight seal and prevent leakage. The bioreactor rests upon an 8-chamber medium reservoir, with each experimental chamber directly overlying its respective medium chamber. The junction between the bioreactor and reservoir is sealed by a gaspermeable membrane (Tegaderm; 3M; St. Paul, MN). An 8channel peristaltic roller pump (Manostat-Carter; Barnant Co.; Barrington, IL) draws medium from the reservoir and administers it to each experimental chamber via $0.89 \mathrm{~mm}$ ID platinum-cured silicone tubing (Cole-Parmer; Vernon-Hills, 
IL). Equipment was sterilized by plasma-phase hydrogen peroxide (Sterrad) processing (bioreactor, screw caps, and reservoir) and steam autoclave (tubing). The apparatus was assembled under sterile conditions in a laminar flow biosafety cabinet. The entire bioreactor was placed in a standard cell-culture incubator $\left(37^{\circ} \mathrm{C}, 95 \%\right.$ humidified air, $5 \% \mathrm{CO}_{2}$ ).

2.2. Scaffold Design. $24 \times 6 \mathrm{~mm}$ cylindrical polyurethane scaffolds (Biomerix; Somerset, NJ) with an average pore size of $200 \mu \mathrm{m}$ and $100 \%$ pore interconnectivity were used in all experiments. Scaffolds were sterilized by ethylene-oxide gas sterilization by the manufacturer and sealed in single-use packets.

2.3. Cell Seeding of Scaffolds. MC3T3-E1 murine preosteoblas-tic cells (Riken Cell Bank; Ibaraki, Japan) were expanded by traditional $2 \mathrm{D}$ static culture at $37^{\circ} \mathrm{C}$ and $5 \% \mathrm{CO}_{2}$. DMEM (Sigma, St. Louis, MO) supplemented with $10 \%$ FBS (Gibco Invitrogen, Carlsbad, CA) was used for this and all subsequent experiments. At confluence, cells were lifted using trypsin, resuspended with the same medium, and used to seed the scaffolds. Passage 3-5 cells were used for all experiments.

Each scaffold was placed in a separate well of a 6well tissue-culture plate for seeding. Due their hydrophobic nature, the scaffolds were compressed to allow sponge-like absorption of the cellular suspension upon release of the compressing force. The scaffolds were seeded at a standard concentration of $4 \times 10^{6}$ cells $/ \mathrm{cm}^{3}$ (scaffolds processed for histology 12 hours after seeding confirmed even distribution of cells throughout the scaffold). After seeding, the scaffolds were surrounded by medium (to prevent desiccation) and placed in the cell-culture incubator overnight to allow cellular adherence. After 24 hours in static culture, cellseeded scaffolds were either place in flow-perfusion culture or continued in static culture.

2.4. Static Culture. For static culture controls, seeded scaffolds were maintained in 6-well tissue culture plates with enough media to cover the scaffold in its entirety $(10 \mathrm{~mL})$. Medium was changed every other day to remove waste products of cell metabolism and provide fresh growth supplements (this protocol was selected following optimization experiments in which static culture of scaffolds in larger volumes of media, such as that used in flow-perfusion, without media changes led to accumulation of waste products and lack of growth supplements in the vicinity of the scaffold), but otherwise the plates were left undisturbed in the cellculture incubator. Scaffolds were harvested at days 0, 2, 4, 6, and 8 ( $n=3$ per time point).

2.5. Dynamic Culture. For dynamic culture, scaffolds were loaded into the bioreactor experimental chambers. Each reservoir chamber was loaded with $80 \mathrm{~mL}$ of fresh medium, which was recycled for the duration of the experiment (maximum 8 days) at a rate of $1.0 \mathrm{~mL} / \mathrm{min}$ as it entered the experimental chamber. This rate was sufficient to provide perfusion and permit chemotransportation, but only generated a fluid shear stress of approximately 0.02 dynes $/ \mathrm{cm}^{2}$.
This fluid shear stress was intentionally selected in order to provide subthreshold mechanotransductive stimulation [4]. The fluid flow rate was based upon optimization studies in which three-dimensional finite element fluid mechanics and mass transport models were developed (data not shown). Scaffolds were harvested at days $0,2,4,6$, and $8(n=3$ per time point).

2.6. Scaffold Histology and Analysis. Scaffolds from each time point were fixed in methanol and paraffin embedded. $5 \mu \mathrm{m}$ transverse sections were stained with hematoxylin and eosin. Sections were viewed on an Olympus BX51 microscope (Olympus; Center Valley, PA). The depth below the scaffold edge was measured using an objective micrometer (Olympus). Sections representing the periphery (top third and bottom third) and core (middle third) of the scaffold were reviewed. The number of cells per $4 \times$ low-power field (LPF) was counted (3 nonconsecutive sections for each region at each time point) by two blinded investigators.

2.7. Cellular Activity. An MTT (3-(4,5-Dimethylthiazol2-yl)-2,5-diphenyltetrazolium bromide) assay was used (Sigma) to measure cellular activity in the scaffolds. In this assay, the conversion of yellow MTT to a purple formazan crystal by viable, metabolically active cells is measured using spectrophotometry. Scaffolds were harvested from static and flow-perfusion culture at 1,2, and 6 days, washed in phosphate-buffered saline (PBS), and homogenized. Each homogenate was subjected to $6 \mathrm{~mL}$ of $0.5 \mathrm{mg} / \mathrm{mL}$ MTT (in PBS) solution and allowed to incubate for 4 hours at $37^{\circ} \mathrm{C}$. Excess MTT solution was then decanted and $5 \mathrm{~mL}$ of extraction solution $(5 \mathrm{~mL}$ isopranolol containing $0.01 \mathrm{~N} \mathrm{HCl})$ was added to the homogenate and allowed to incubate at 20 minutes at $37^{\circ} \mathrm{C} .100 \mu \mathrm{L}$ aliquots of the resulting supernatant were then added to a 96-well plate and the absorbance at $570 \mathrm{~nm}$ was determined using spectrophotometry. Cell activity was then expressed as the absorbance at $570 \mathrm{~nm}$ per gram of scaffold.

2.8. Statistical Analysis. All data are expressed as mean \pm standard error of the mean. Data were analyzed with a two-tailed Student's $t$-test assuming unequal variance using SigmaStat (SPSS Science; Chicago, IL). Values of $P<.05$ were considered significant.

\section{Results}

3.1. Cells Are Distributed Uniformly after Seeding. Scaffolds harvested 12 hours after seeding demonstrated a homogenous distribution of cells in pores throughout the scaffold (Figure 2). At this early time point, cell density in the periphery $(109.3 \pm 5.5$ cells/LPF $)$ and core $(106.8 \pm 3.9$ cells/LPF $)$ was not significantly different $(P=.67)$.

3.2. Cusp of Viability in Static Culture Is 4 Days. Scaffolds in static culture had a slow, but significant decline in the peripheral cell density over the course of the experiment (Figure 3). Compared to the initial seeding density (109.3 \pm 5.5 cells/LPF), there were $106.7 \pm 2.0$ cells/LPF $(97.6 \%$ of the 


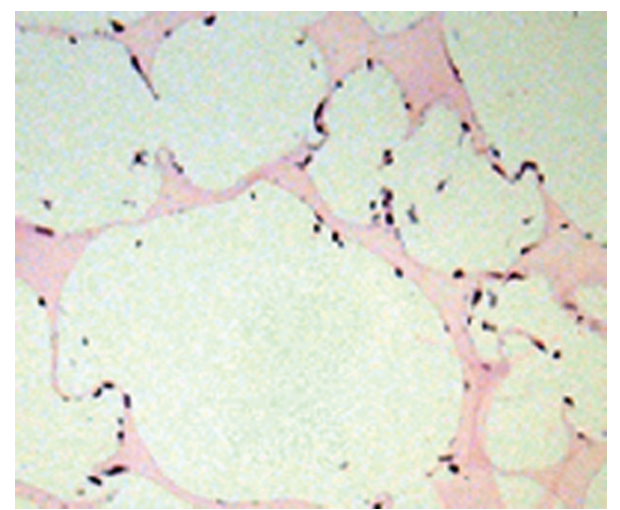

FIGURE 2: Photomicrograph $(100 \times)$ highlighting the uniform cell distribution within the scaffold. All pores throughout the scaffolds have a similar appearance 12 hours after seeding.

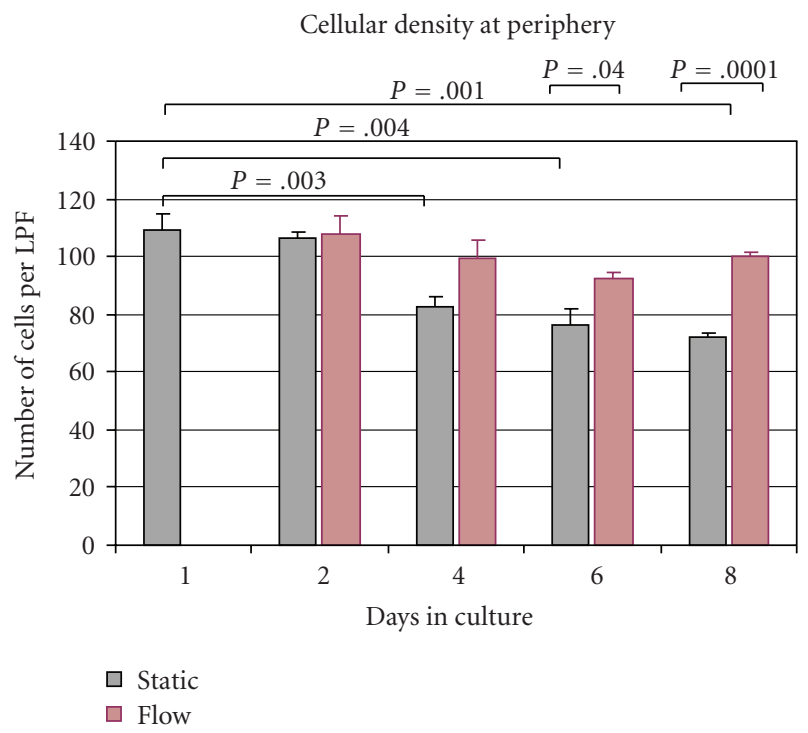

FIGURE 3: Static cultured scaffolds had a 34\% reduction in cellular viability at the periphery over the experimental period. In contrast, dynamic culture resulted in an $8.3 \%$ decrease in cell viability.

initially seeded cells) at day $2(P=.4), 82.3 \pm 3.8$ cells/LPF $(75.2 \%)$ at day $4(P=.003), 76.3 \pm 5.2$ cells/LPF $(69.8 \%)$ at day $6(P=.004)$, and $72.3 \pm 1.2$ cells/LPF $(66.1 \%)$ at day $8(P=.001)$. Cell density in the periphery was similar to a depth of approximately $2 \mathrm{~mm}$ from the superior and inferior surface of the scaffold. There was no statistical difference in cell density between superior and inferior portions of the periphery at any time point (data not shown).

In marked contrast to the peripheral crust (outer $2 \mathrm{~mm}$ ) of the scaffold, the core (central $2 \mathrm{~mm}$ ) of these same scaffolds exhibited a rapid decline in cell density (Figure 4(a)). Within the core, cell density was $70.3 \pm 5.5$ cells/LPF $(64.3 \%$ remaining of the initially seeded cells) at day $2(P=.003)$, $39.0 \pm 4.4(35.7 \%)$ at day $4(P=.001), 4.67 \pm 1.8(4.2 \%)$ at day $6(P<.001)$, and $0.25 \pm 0.25(0.2 \%)$ at day $8(P<.001)$. The cusp of viability for cells in the core was day 4 ; between days 4 and 6 , there was a dramatic $88 \%$ reduction $(P=.002)$ in cell density (Figure 4(b)).

The metabolic activity of cells in static culture also declined over the course of the experiment (Figure 5). MTT cell assay demonstrated that cellular activity in scaffolds in static culture declined to $74 \%$ of initial activity $(0.39 \pm 0.04 \mathrm{OD} / \mathrm{g})$ at day 2 and $70 \%$ of initial activity $(0.37 \pm 0.04 \mathrm{OD} / \mathrm{g})$ at day 6 . The change in metabolic activity was significant by day $6(P=.025)$. In static culture, metabolically active cells were limited to the periphery of the scaffold.

3.3. Flow-Perfusion Culture Promotes Cellular Viability in the Scaffold Core. Similar to static culture, scaffolds placed in flow-perfusion had a peripheral cell density of $108.0 \pm 5.9$ cells/LPF ( $98.9 \%$ of the initially seeded cells) at day $2,99.3 \pm 6.1(90.9 \%)$ at day $4,92.7 \pm 1.8(84.9 \%)$ at day 6 , and $100.0 \pm 1.5(91.5 \%)$ at day 8 (Figure 3 ). Pairwise comparisons demonstrated a significantly greater number of cells in the periphery of scaffolds placed in flow-perfusion on days $6(P=.04)$ and $8(P=.0001)$. Similar to static culture samples, there was no difference in cellular density between the inferior and superior thirds of the scaffold (data not shown).

In marked contrast to static culture, scaffolds placed in flow-perfusion maintained significantly higher cell density within the core of the scaffold (Figures 6(a) and 6(b)). Core cell density was $103.7 \pm 2.3$ cells/LPF $(94.9 \%$ of the initially seeded cells) at day 2, $80 \pm 5.5$ cells/LPF $(73.2 \%)$ at day $4,83.5 \pm 1.5$ cells/LPF $(76.4 \%)$ at day 6 , and $81.7 \pm$ 6.4 cells/LPF $(74.7 \%)$ at day 8 (Figure $4(\mathrm{a})$ ). The differences in core cell density between static and flow-perfusion culture were statistically significant at days $2(P=.01), 4(P<.001)$, $6(P=.006)$, and $8(P=.002)$.

The metabolic activity of cells in flow-perfusion culture significantly increased over the course of the experiment (Figure 5). MTT cell assay demonstrated that cellular activity in scaffolds in dynamic culture increased 125\% (0.66 \pm $0.03 \mathrm{OD} / \mathrm{g})$ at day 2 and 285\% (0.37 $\pm 0.04 \mathrm{OD} / \mathrm{g})$ at day 6 compared to the initial activity. The increase in metabolic activity in flow-perfusion was not only significantly greater than the initial activity (day $2, P=.035$; day $6, P=$ .018 ), but it was also significantly greater than the activity in static culture at similar time (day 2, $P=.002$; day 6 , $P=.006)$.

\subsection{Core : Periphery Ratio Is Maintained in Flow-Perfusion} Culture. To account for the possibility of confounding shear stress (despite subthreshold mechanotransductive flow) or differences in cell distribution in static versus dynamic culture, the core: periphery ratio was calculated (Figure 6(c)). In static culture, the ratio was $0.66 \pm 0.05$ at day $2,0.48 \pm 0.07$ at day $4,0.06 \pm 0.02$ at day 6 , and $0.004 \pm 0.004$ at day 8 . In contrast, flow perfusion maintained the core : periphery ratio to $0.97 \pm 0.07$ at day $2,0.82 \pm 0.10$ at day $4,0.61 \pm 0.30$ at day 6 , and $0.81 \pm 0.07$ at day 8 . The differences in core : periphery ratio of static versus dynamic were statistically significant: day $2(P=.004)$, day $4(P=.005)$, day $6(P=.01)$, and day $8(P=.004)$. 


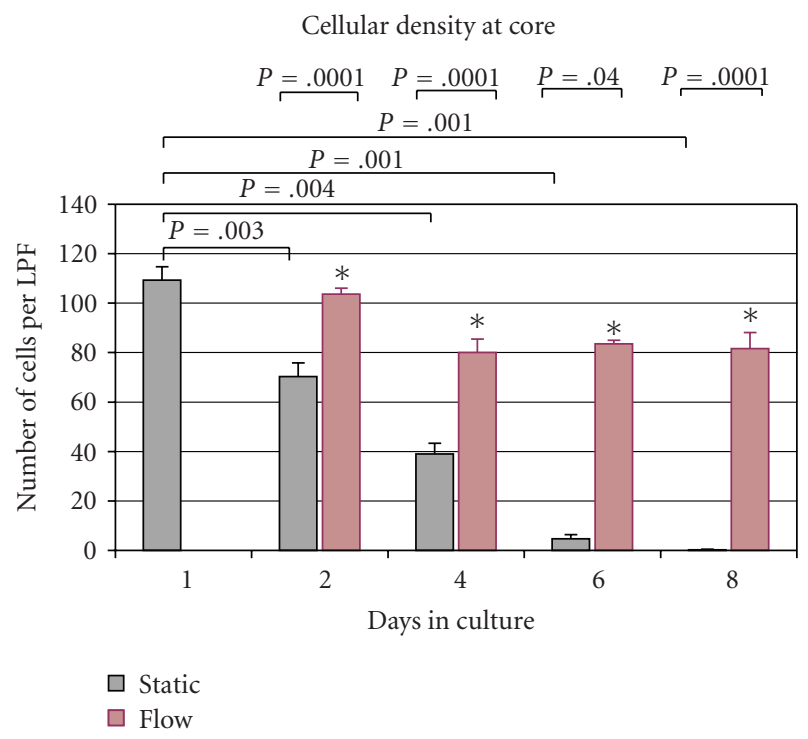

(a)

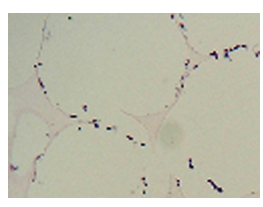

1

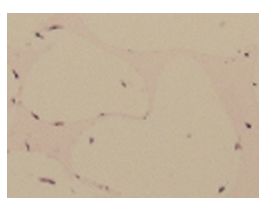

4

Days in culture

(b)

Figure 4: (a) Cell density in the core exhibited a rapid decline in scaffolds cultured in static conditions. In contrast, core cell density was maintained in scaffolds treated with flow-perfusion culture. (b) Photomicrograph of MC3T3-E1 cells in the core of a polyurethane scaffold in static culture. The cusp of viability for cells in the core lies between day 4 and day 6 for samples in static culture. Between these time points, there was an $88 \%$ reduction in cell density.

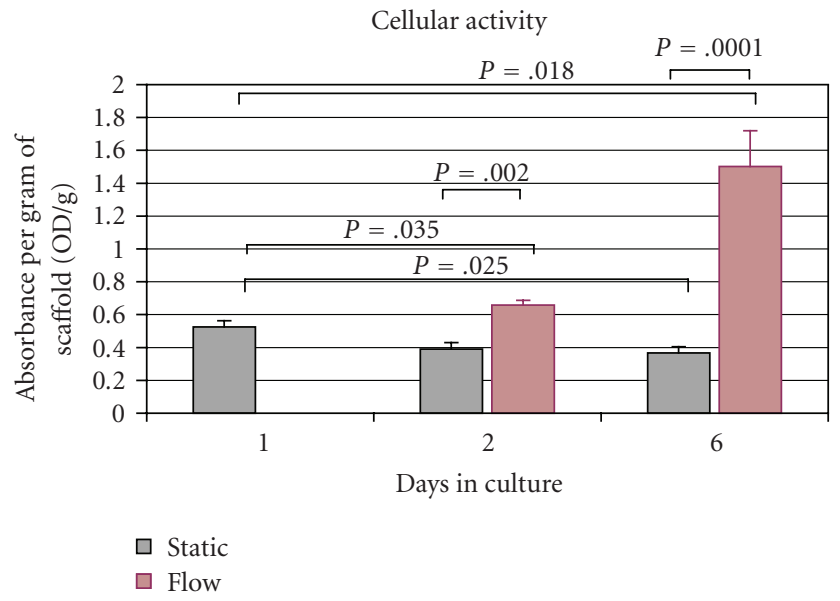

Figure 5: Cellular activity in static and flow-perfusion culture. MTT assay demonstrates no significant change in static culture at day 2. However, metabolic activity increased significantly in cells in flow-perfusion at days 2 and 6 . Metabolic activity also significantly decreased in static culture at day 6 .

\section{Discussion}

The ability to maintain cell viability in vitro in thick $3 \mathrm{D}$ scaffolds has important implications for tissue engineering. For example, attempts at 3D in vitro bone culture without adequate chemotransportation have invariably shown an inverse relationship between construct thickness and cellular survival $[2,12]$. This inverse relationship is due to a decline in nutrients and accumulation of waste products in the core of the construct during bone matrix deposition and mineralization.

Traditional cell-culture methods are not well suited to the maintenance of 3D tissue-engineered constructs due to the inherent limitation in chemotransportation. In our study, we demonstrated that, under static culture conditions, cells in the peripheral crust up to $2 \mathrm{~mm}$ deep from the external surface were able to survive by static diffusion, a finding that is in agreement with other published accounts describing survival to a depth of 1-2 mm $[3,13]$. However, we noted a linear decline in core cell density over time, with less than $5 \%$ of cells remaining after 6 days of static culture. These observed temporospatial differences in cell density with static culture may be explained by two phenomena associated with poor nutrient diffusion: (1) death of cells in the interior core of the scaffold, and (2) chemotaxis of cells from the core toward the periphery [14].

In an effort to support 3D tissue-engineered constructs, various bioreactor systems have been designed, including the spinner flask and the rotational bioreactor. However, because these designs simply move fluid across the exterior of the scaffold, chemotransportation to the interior is not guaranteed. The flow-perfusion bioreactor differs in that it ensures nutrient transport by perfusing medium through the interconnected pores of the scaffold [11]. Moreover, the design allows the investigator to control mechanical forces; the pump speed may be set from 3.5 to 200 revolutions per minute for perfusion ranging from 0.26 to $14.8 \mathrm{mls} / \mathrm{min}$, replicating shear stress from $0.01-10$ dynes $/ \mathrm{cm}^{2}$-physiologic in vivo range for osteocytes $\left(8-30\right.$ dynes $\left./ \mathrm{cm}^{2}\right)$ [4].

We found that dynamic culture using a flow perfusion bioreactor significantly improved core cell activity and density compared to static culture. After a slight initial decrease in cell density between days 2 and 4, core cell density in flow perfusion was maintained throughout the course of the experiment at approximately $80 \%$ of the initial seeding density. Likewise, the core:periphery ratio, which compensates for differences in cell distribution and potential effects of fluid shear stress between culture systems, was steady (approximately $0.6-0.8$ ) from day 2 to day 8 .

While originally designed to improve chemotransportation, the flow-perfusion bioreactor has the ability to generate fluid-shear forces at the cellular level at higher rates of medium flow [15]. Although fluid shear forces were intentionally kept subphysiologic (i.e., less than 8 dynes $/ \mathrm{cm}^{2}$ ), to reduce/eliminate the effects mechanical stimulation during this study (to concentrate on the effect of chemotransportation, and not mechanical stimulation), other studies have suggested that fluid shear forces may be useful for bone or vascular tissue engineering $[11,16,17]$. 


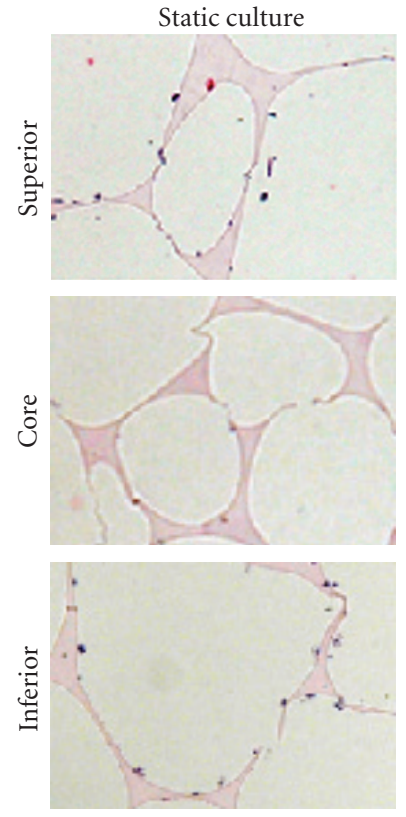

(a)

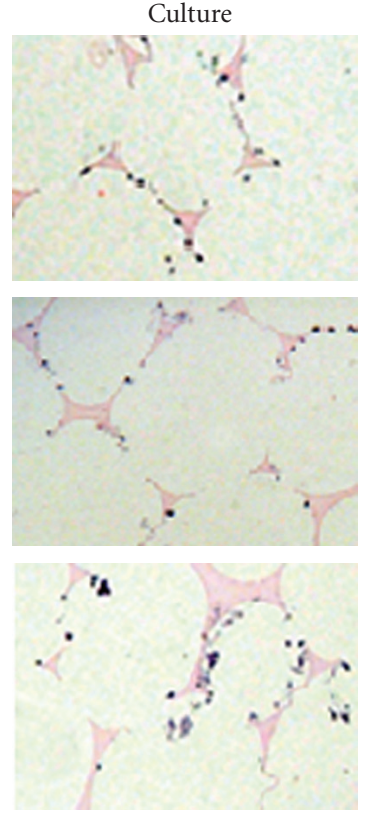

(b)

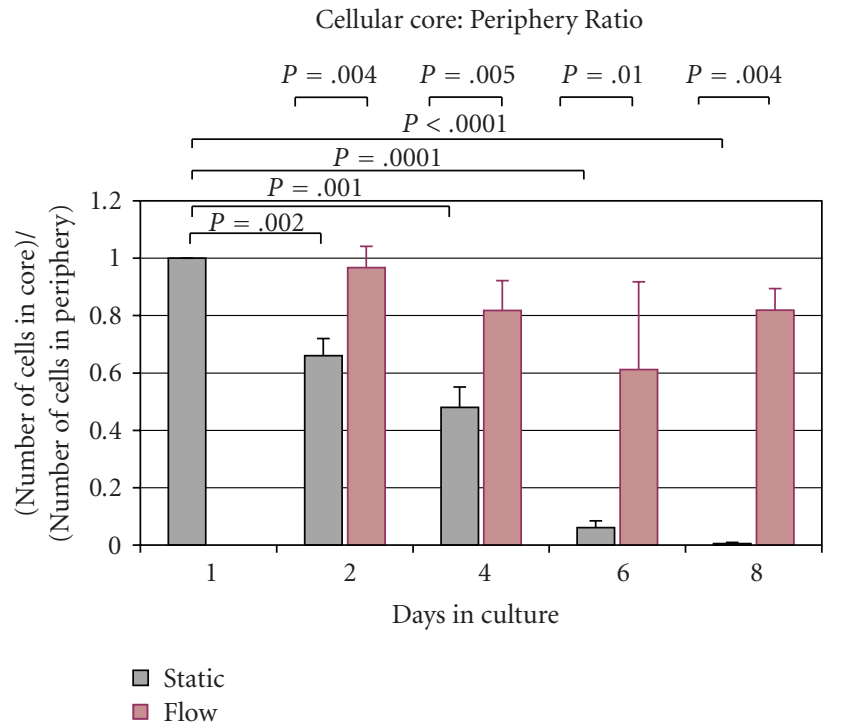

(c)

FIGURE 6: (a) Dividing the scaffold into thirds, the core of static cultured scaffolds was nearly void of cells by day 4 . Cell density was preserved in the peripheral crust (superior and inferior thirds). (b) Scaffolds exposed to flow-perfusion culture exhibit a near homogenous cell density in all areas of the scaffold, with evident cell viability in the core, in contrast to the static cultured samples. (c) To account for the possibility of confounding shear stress or differences in cell proliferation in dynamic versus static culture, the core : periphery ratio was calculated.

\section{Conclusions}

Ultimately, in order to solve clinical problems, engineered bony tissue must be fashioned into 3D patient-specific sizes and shapes. Custom-printed scaffolds (e.g., those designed from actual patient computed tomography data) are too thick to survive by nonpenetrating chemotransportation. However, the flow-perfusion bioreactor is in theory a scalable technology that should support porous scaffolds of any thickness. This study demonstrates that thick $(>6 \mathrm{~mm}) 3 \mathrm{D}$ constructs are sustainable using a flow-perfusion bioreactor. Future work will concentrate on computational modeling of the fluid dynamics and mass transport using specific scaffold designs, and on analysis of cellular proliferation, differentiation, and organization within the construct.

\section{Acknowledgments}

This work was graciously supported in part by the 2008 Alpha Omega Alpha Carolyn L. Kuckein Research Fellowship (to the first author); the 2008 American Medical Association Seed Grant Research Program (to the first author); the 2007 Lyndon Peer Research Fellowship, administered by the Plastic Surgery Educational Foundation (to the second author); the 2007 Bernd Spiessel Research Award, administered by the American Society of Maxillofacial Surgery through the courtesy of Synthes CMF (to the second author); the 2007 Plastic Surgery Educational Foundation Basic Science Research Grant (to the second author); the 2008 Association of Academic Plastic Surgeons Academic Scholar Award (to the sixth author). The authors would like to express their gratitude to James Vertolli and Umberto Allori for their creative input and tireless efforts in the design and manufacturing of our bioreactor. The authors have no commercial or financial associations that might create a conflict of interest with the information presented in this manuscript.

\section{References}

[1] P. V. Guillot, W. Cui, N. M. Fisk, and D. J. Polak, "Stem cell differentiation and expansion for clinical applications of tissue engineering," Journal of Cellular and Molecular Medicine, vol. 11, no. 5, pp. 935-944, 2007.

[2] J. van den Dolder, G. N. Bancroft, V. I. Sikavitsas, P. H. M. Spauwen, J. A. Jansen, and A. G. Mikos, "Flow perfusion culture of marrow stromal osteoblasts in titanium fiber mesh," Journal of Biomedical Materials Research, vol. 64, no. 2, pp. 235-241, 2003.

[3] V. I. Sikavitsas, G. N. Bancroft, and A. G. Mikos, "Formation of three-dimensional cell/polymer constructs for bone tissue engineering in a spinner flask and a rotating wall vessel bioreactor," Journal of Biomedical Materials Research, vol. 62, no. 1, pp. 136-148, 2002.

[4] S. Weinbaum, "A model for the excitation of osteocytes by mechanical loading-induced bone fluid shear stresses," Journal of Biomechanics, vol. 27, no. 3, pp. 339-360, 1994.

[5] S. P. Fritton, K. J. McLeod, and C. T. Rubin, "Quantifying the strain history of bone: spatial uniformity and self-similarity of low-magnitude strains," Journal of Biomechanics, vol. 33, no. 3, pp. 317-325, 2000. 
[6] G. Vunjak-Novakovic, I. Martin, B. Obradovic, et al., "Bioreactor cultivation conditions modulate the composition and mechanical properties of tissue-engineered cartilage," Journal of Orthopaedic Research, vol. 17, no. 1, pp. 130-138, 1999.

[7] G. Vunjak-Novakovic, B. Obradovic, I. Martin, P. M. Bursac, R. Langer, and L. E. Freed, "Dynamic cell seeding of polymer scaffolds for cartilage tissue engineering," Biotechnology Progress, vol. 14, no. 2, pp. 193-202, 1998.

[8] J. Glowacki, S. Mizuno, and J. S. Greenberger, "Perfusion enhances functions of bone marrow stromal cells in threedimensional culture," Cell Transplantation, vol. 7, no. 3, pp. 319-326, 1998.

[9] L. E. Freed, N. Pellis, N. Searby, et al., "Microgravity cultivation of cells and tissues," Gravitational and Space Biology Bulletin, vol. 12, no. 2, pp. 57-66, 1999.

[10] L. E. Freed, G. Vunjak-Novakovic, and R. Langer, "Cultivation of cell-polymer cartilage implants in bioreactors," Journal of Cellular Biochemistry, vol. 51, no. 3, pp. 257-264, 1993.

[11] G. N. Bancroft, V. I. Sikavitsas, and A. G. Mikos, "Design of a flow perfusion bioreactor system for bone tissue-engineering applications," Tissue Engineering, vol. 9, no. 3, pp. 549-554, 2003.

[12] T. A. Owen, M. Aronow, V. Shalhoub, et al., "Progressive development of the rat osteoblast phenotype in vitro: reciprocal relationships in expression of genes associated with osteoblast proliferation and differentiation during formation of the bone extracellular matrix," Journal of Cellular Physiology, vol. 143, no. 3, pp. 420-430, 1990.

[13] L. G. Griffith and M. A. Swartz, "Capturing complex 3D tissue physiology in vitro," Nature Reviews Molecular Cell Biology, vol. 7, no. 3, pp. 211-224, 2006.

[14] A. S. Goldstein, T. M. Juarez, C. D. Helmke, M. C. Gustin, and A. G. Mikos, "Effect of convection on osteoblastic cell growth and function in biodegradable polymer foam scaffolds," Biomaterials, vol. 22, no. 11, pp. 1279-1288, 2001.

[15] V. I. Sikavitsas, G. N. Bancroft, H. L. Holtorf, J. A. Jansen, and A. G. Mikos, "Mineralized matrix deposition by marrow stromal osteoblasts in 3D perfusion culture increases with increasing fluid shear forces," Proceedings of the National Academy of Sciences of the United States of America, vol. 100, no. 25, pp. 14683-14688, 2003.

[16] S. Li, N. F. Huang, and S. Hsu, "Mechanotransduction in endothelial cell migration," Journal of Cellular Biochemistry, vol. 96, no. 6, pp. 1110-1126, 2005.

[17] K. Yamamoto, T. Takahashi, T. Asahara, et al., "Proliferation, differentiation, and tube formation by endothelial progenitor cells in response to shear stress," Journal of Applied Physiology, vol. 95, no. 5, pp. 2081-2088, 2003. 

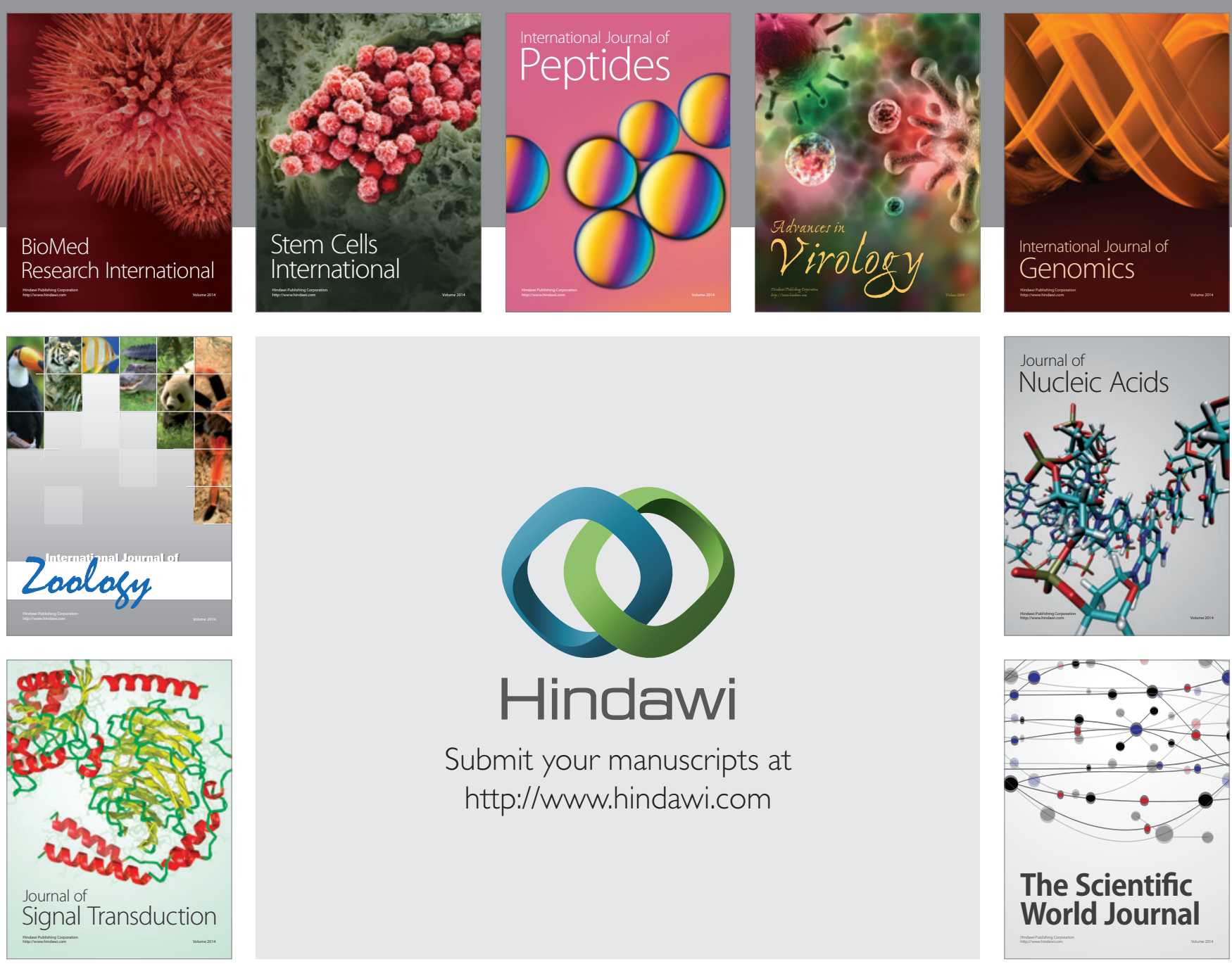

Submit your manuscripts at

http://www.hindawi.com
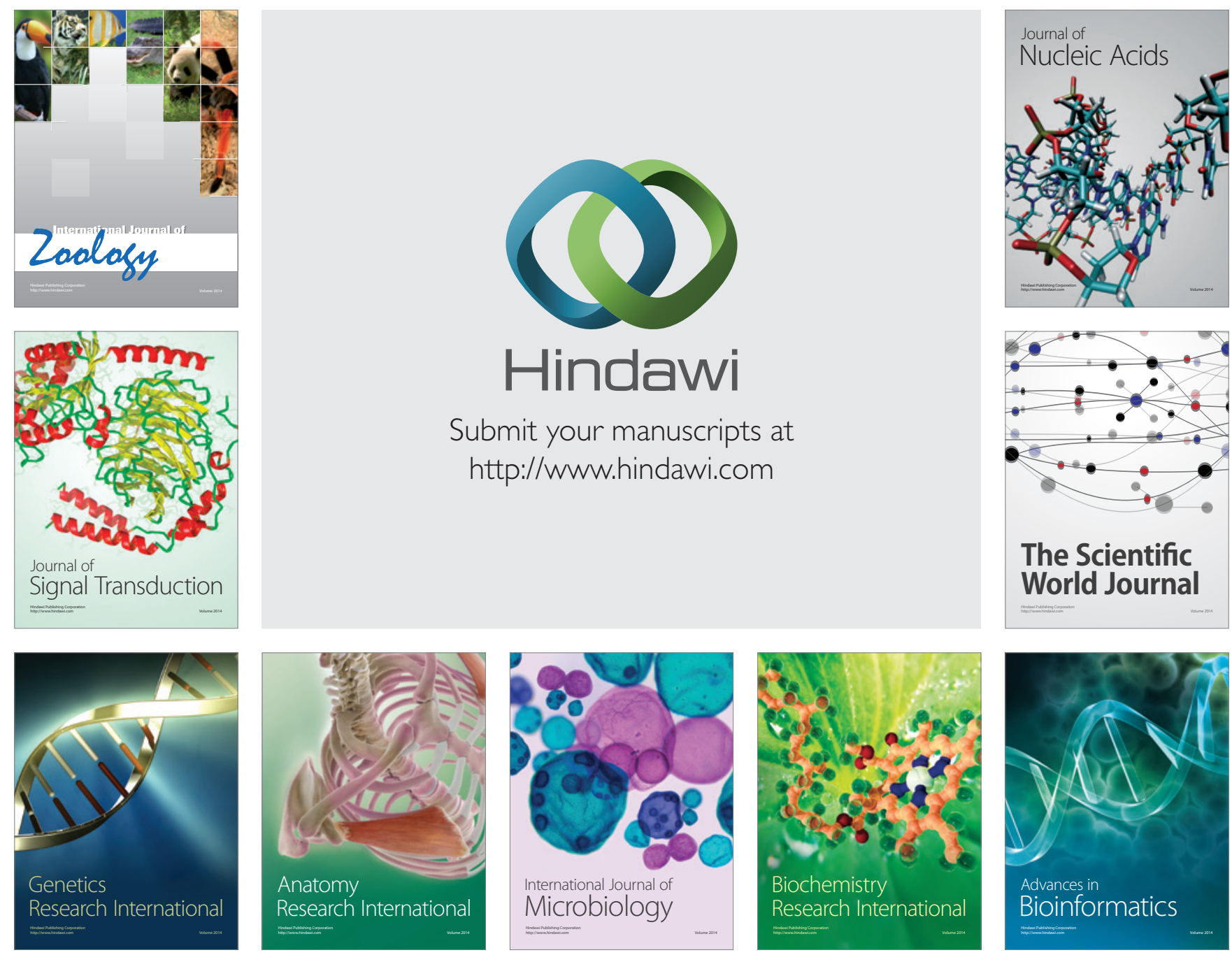

The Scientific World Journal
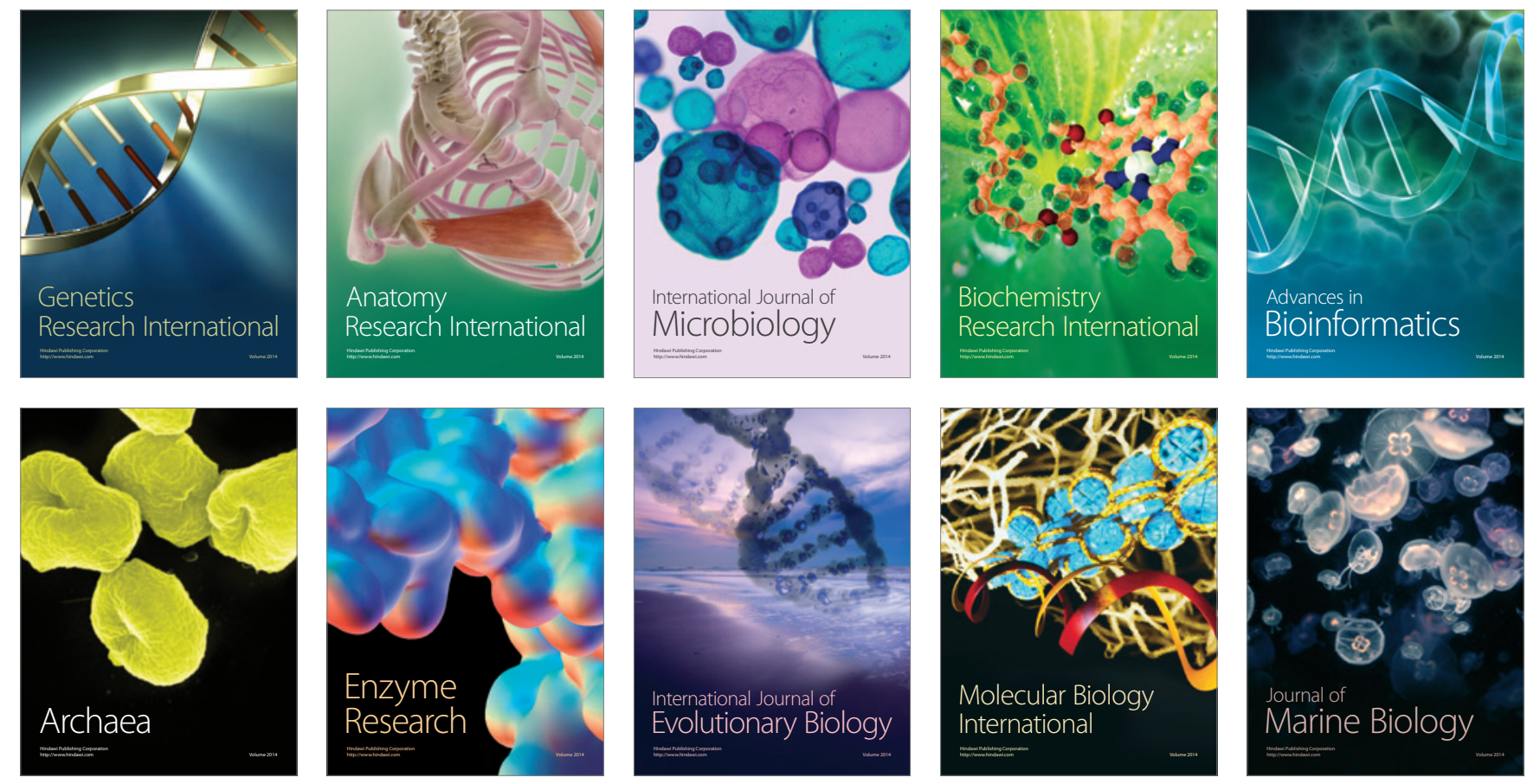